\title{
Immunomodulatory Effectiveness of Fish Oil and omega-3 Fatty Acids in Human Non-melanoma Skin Carcinoma Cells
}

\author{
Khurram Rehman ${ }^{1}$, Mohd Cairul Iqbal Mohd Amin ${ }^{1}, \mathrm{Ng}$ Pei Yuen ${ }^{2}$ and \\ Mohd Hanif Zulfakar ${ }^{1 *}$ \\ ${ }^{1}$ Center for Drug Delivery Research, Faculty of Pharmacy, Universiti Kebangsaan MALAYSIA \\ ${ }^{2}$ Drug \& Herbal Research Centre, Faculty of Pharmacy, Universiti Kebangsaan MALAYSIA
}

\begin{abstract}
Fish oil is composed of various fatty acids among which omega-3 fatty acids are considered as most beneficial. The effects of fish oil on the activity of a topical anticancer drug, imiquimod, and the immunomodulatory activity of omega-3 fatty acids was investigated in human basal and squamous cell carcinoma cell lines. Imiquimod-fish oil mixture exhibited higher carcinoma cell growth inhibition and immunomodulatory activity than imiquimod alone, especially against squamous cell carcinoma cells. Omega-3 fatty acids exhibited growth inhibition of both basal cell and squamous cell carcinoma cell lines and modulated the immune response. Omega-3 fatty acids of fish oil serve as inducers of interleukin-10, an anti-inflammatory cytokine, and as suppressors of interleukin-6 and tumor necrosis factor-alpha, which not only depress tumor growth but also adequately control the inflammatory side effects of imiquimod. Thus, imiquimod administration with fish oil could be beneficial for inhibition of non-melanoma skin carcinoma cells but further in vivo studies are needed to understand their role in skin cancer.
\end{abstract}

Key words: fish oil, basal cell carcinoma, eicosapentaenoic acid, docosahexaenoic acid, squamous cell carcinoma, immunomodulatory

\section{Introduction}

Non melanoma skin carcinoma is the most common malignant tumor in humans that deriving from non-keratinizing cells and originates in the basal and squamous layers of the epidermis, most commonly known as basal cell carcinoma (BCC) and squamous cell carcinoma (SCC) respectively. Imiquimod is an immune response modifier ${ }^{1)}$ and has been widely prescribed by dermatologists as a topical treatment, especially for curing skin conditions such as actinic keratosis, skin warts, and carcinoma ${ }^{2)}$. Conversely, it is also known for its inflammatory-like side effects, such as severe skin inflammation ${ }^{3,4)}$. In our earlier work, we demonstrated an interaction between fish oil and imiquimod ${ }^{5)}$ to explain observations seen in the delivery of imiquimod from fish oil-based gel system. Through computational molecular modeling, the possibility of a complex formation between imiquimod and EPA fatty acid of fish oil through a $\pi-\sigma$ interaction between the aromatic ring of the drug and a longchain fatty acid was confirmed. Such interactions play an important role in biological systems and molecular recogni- tion $^{6,7)}$ and may also help in reducing drug-related inflammatory side effects ${ }^{5}$.

Fish oil is extracted from tissues of marine or fresh water fish and is a primary source of omega-3 fatty acids, especially eicosapentaenoic acid (EPA) and docosahexaenoic acid (DHA). The composition of omega-3 fatty acids depends on the body part of fish, method employed to extract the fish oil and source of fish. The omega-3 fatty acids, eicosapentaenoic acid (EPA) and docosahexaenoic acid (DHA) are associated with many beneficial pharmacological activities in various diseases, such as cardiovascular disease $^{8)}$, breast cancer ${ }^{9)}$, and pancreatic cancer ${ }^{10)}$, and are also considered anti-inflammatory agents ${ }^{11,12)}$. The aim of this study was to determine the immunomodulatory effectiveness of imiquimod (IMQ), imiquimod-fish oil mixture (1:1 ratio by weight) combination (IMQ-FO), EPA, and DHA against non-melanoma carcinoma cell lines.

Human squamous cell carcinoma cell line A431 $\left(\mathrm{ATCC}^{\circledR}\right.$ CRL-1555) and human basal cell carcinoma cell line TE 354.T $\left(\right.$ ATCC $^{\circledR}$ CRL-7762) cell line were used in this study.

\footnotetext{
*Correspondence to: Mohd Hanif Zulfakar, Center for Drug Delivery Research, Faculty of Pharmacy, Universiti Kebangsaan MALAYSIA

E-mail: hanifzulfakar@ukm.edu.my

Accepted December 6, 2015 (received for review November 6, 2015)

Journal of Oleo Science ISSN 1345-8957 print / ISSN 1347-3352 online

http://www.jstage.jst.go.jp/browse/jos/ http://mc.manusriptcentral.com/jjocs
} 
Human squamous cell carcinoma cell line A431 has been widely studied in different fields of biomedical research ${ }^{13-17)}$, whereas very little work could be found on basal cell carcinoma cell line TE 354. $\mathrm{T}^{18)}$. The study was divided into two sections, first the inhibitory ability of the IMQ, IMQ-FO, EPA and DHA on carcinoma cell line growth was determined through Alamar ${ }^{\circledR}$ blue assay and second, the immunomodulatory effects of all these compounds were determined through immunoassay for cytokines such as interleukin-6 (IL6), interleukin-10 (IL10), and tumor necrosis factor-alpha $(\mathrm{TNF}-\alpha)$. These cytokines were analyzed because of their relationship with non-melanoma skin cancer, inflammation, and imiquimod itself ${ }^{19-21)}$.

\section{Materials and methodology \\ 2.1 Materials}

Blackmores Fish Oil (42\% EPA and 21\% DHA)was purchased from Blackmores (Warriewood, Australia). Supelco ${ }^{\circledR}$ 37 FAME mix, methyl eicosapentaenoate, methyl decosahexaenoate analytical standards were purchased from Sigma-Aldrich (St. Louis, USA). Dulbecco's modified eagle medium (DMEM, high glucose, with L-glutamine and phenol red, no HEPES, no sodium pyruvate), fetal bovine serum (FBS), trypsin-EDTA $0.25 \%$ with phenol red, penicillin-amphotericin B(Pen-Strep $1000 \mathrm{U} / \mathrm{mL}$ ), phosphate buffer solution (PBS 1X), and Alamar ${ }^{\circledR}$ blue were obtained from Gibco ${ }^{\circledR}$ (Malaysia). Imiquimod (R837) and trypan blue were purchased from Invivogen(USA). Corning cell culture flasks, 24- and 96-well plates, disposable pipettes, dimethylsulfoxide(DMSO), hydrochloric acid, acetone, absolute alcohol, and ethanol were obtained from Fisher Scientific (Waltham, USA). Human squamous cell carcinoma cell line A431 (ATCC ${ }^{\circledR}$ CRL-1555) and human basal cell carcinoma cell line TE 354.T (ATCC ${ }^{\circledR}$ CRL-7762) were obtained from American Type Culture Collection (ATCC) (Singapore) and human ELISA kits for IL6, IL10 and TNF- $\alpha$ were obtained from Science cell(USA).

\subsection{Determination of Fish Oil Composition}

Determination of fatty acid composition in fish oil was conducted using a gas chromatography (GC) 2010 instrument (Shimadzu Corp., Kyoto, Japan) with a flame ionization detector(FID). The injector temperature was maintained at $250^{\circ} \mathrm{C}$, and the detector temperature was maintained at $275^{\circ} \mathrm{C}$. The temperature of the column was maintained at $180^{\circ} \mathrm{C}$ for 2 minutes, and then it gradually increased to $240^{\circ} \mathrm{C}$ at a rate of $4^{\circ} \mathrm{C} / \mathrm{min}$. Nitrogen was used as a carrier gas with a flow rate of $60 \mathrm{~cm} / \mathrm{s}$. Fatty acid methyl esters (FAMEs) were prepared by adding $0.5 \mathrm{~mL}$ of $0.5 \mathrm{M}$ anhydrous methanol-sodium methoxide solution was added to a solution of $5 \mathrm{mg}$ of fish oil in $1 \mathrm{~mL}$ of hexane, in centrifuge tube(vortexed for 1 minute). Aliquots of $1 \mu \mathrm{L}$
FAME was injected into the highly polar cyanosiloxane column, SP-2380 (30 mm $\times 0.25 \mathrm{~mm} \times 0.20 \mu \mathrm{m}$ film thickness) from Supelco (USA). Individual peaks in fish oil were identified by comparing their retention time with those of the retention time of Supelco analytical standards (SigmaAldrich). Individual EPA and DHA concentration was calculated using the peak areas of the fatty acid that appear in the chromatogram as the relative percentage of the total peak areas of all the fatty acids in the oil sample.

\subsection{Cell Viability}

Cell viability was performed by Alamar $^{\circledR}$ blue assay. Human squamous cell carcinoma (SCC) cell lines A431 and basal cell carcinoma (BCC) TE 354.T were maintained in DMEM supplemented with $10 \%$ FBS. For the viability test, cells were seeded in 96-well plates at a density of $2.5 \times 10^{4}$ cells/mL and left to incubate overnight. Cells were treated with serial dilutions of IMQ, IMQ-FO, EPA, and DHA for 24 hours and then Alamar ${ }^{\circledR}$ blue assay was performed to determine cell viability.

\subsection{Immunomodulatory assays on human skin carcinoma cell lines}

Enzyme-linked immunosorbent assays for IL6, IL10, and TNF- $\alpha$ were performed on BCC and SCC cell lines and the immunomodulatory effectiveness of IMQ, IMQ-FO, EPA, and DHA on these cell lines was determined. The cells were seeded in a 24 -well plate using the technique described above with a density of $3 \times 10^{5}$ cells $/ \mathrm{mL}$. The plates were prepared under aseptic conditions and left to incubate overnight at $37^{\circ} \mathrm{C}$ and $5 \% \mathrm{CO}_{2}$. The next day, cells were treated with IMQ, IMQ-FO, EPA, and DHA solutions and the plate was again incubated for $24 \mathrm{~h}$. After incubation, the cell supernatant was collected and stored at $-20^{\circ} \mathrm{C}$ prior to the immunosorbent assay. In vitro analysis for quantitative measurement of human IL6, IL10, and TNF- $\alpha$ in carcinoma cell lines was performed using ELISA. All reagents were prepared according to the manufacturer's protocol before performing the assay.

\subsection{Statistical analysis}

Data are tabulated using Microsoft Excel 2015 (Microsoft Corporation, Seattle), and statistical analysis performed on IBM SPSS Statistics ver. 20 (IBM Corporation, New York). All results are expressed as mean \pm SD. Significant differences in study groups were evaluated using paired t-test. $\mathrm{P}$ value less than $0.05(p<0.05)$ was considered significant.

\section{Results and discussion}

\subsection{Determination of Fish Oil Composition}

The GC analysis was conducted to analyze the composition of Blackmores ${ }^{\circledR}$ fish oil and its stated label claim (42\% 
Table 1 Composition of fatty acids present in Blackmores ${ }^{\circledR}$ fish oil.

\begin{tabular}{lcc}
\hline \multicolumn{1}{c}{ Fatty acid } & Retention time (min) & Percentage (\%) \\
\hline Myristic acid 14:0 & 2.95 & 0.41 \\
Palmitic acid 16:0 & 3.58 & 1.43 \\
Stearic acid 18:0 & 4.54 & 0.62 \\
Oleic acid 18:1 & 4.94 & 10.16 \\
Linoleic acid 18:2 & 5.58 & 1.63 \\
Linolenic acid 18:3 & 6.40 & 3.44 \\
Arachidonic acid 20:4 & 8.30 & 2.31 \\
Eicosapentaenoic acid 20:5 & 9.46 & 40.68 \\
Docosahexaenoic acid 22:6 & 12.16 & 23.08 \\
\hline
\end{tabular}

EPA and 21\% DHA). The fish oil chromatograph revealed number of fatty acid peaks that were identified through their retention time with sigma analytical standards. The retention time for EPA and DHA was found to be $9.46 \mathrm{~min}$ and $12.16 \mathrm{~min}$. The percentage of the EPA and DHA were calculated by the normalization method and the fish oil sample was found to contain $40.86 \% \mathrm{EPA}$ and $23.08 \%$ DHA. Apart from EPA and DHA, other fatty acids found in fish oil and their retention time is given in Table 1. Analysis of fish oil composition was necessary in order to find out exact concentration of omega-3 fatty acids present inside it. Later in the study, this fish oil was used to prepare mixtures with the imiquimod and analyzed for its effectiveness against the carcinoma cell lines.

\subsection{Cell viability assay of SCC and BCC cell lines}

Cytotoxcity study was conducted to determine the effectiveness of IMQ, IMQ-FO combination, and omega-3 fatty acids of fish oil (EPA and DHA) on skin carcinoma cell lines and to determine the concentration-dependent response to the cell viability. It was observed that in SCC, IMQ-FO combination (only 150, 200 and $250 \mu \mathrm{g} / \mathrm{mL}$ concentrations) exhibited higher reduction in carcinoma cell viability as compared to $\operatorname{IMQ}(p<0.05$, paired t-test) (Fig. 1). To analyze the omega-3 fatty acids influence on SCC cell viability, carcinoma cells treated with EPA and DHA dilutions exhibited concentration dependent decline in cell viability of carcinoma cells. Thus it could be suggested that these fish oil omega-3 fatty acids were responsible for reduction in cell viability of SCC cell lines when treated with IMQ-FO combinations (Fig. 1). In case of BCC, cells treated with IMQ-FO and IMQ dilutions showed similar inhibition to cell viability with very minimal difference was reported (apart from 50 and $100 \mu \mathrm{g} / \mathrm{mL}$ concentrations). Both EPA and DHA also showed their effectiveness against BCC, and exhibited concentration dependent decline in cell viability (Fig. 2). The cell viability studies suggested that the omega-3 fatty acids (pure EPA and DHA) are more potent than fish oil for their immunomodulatory activity against
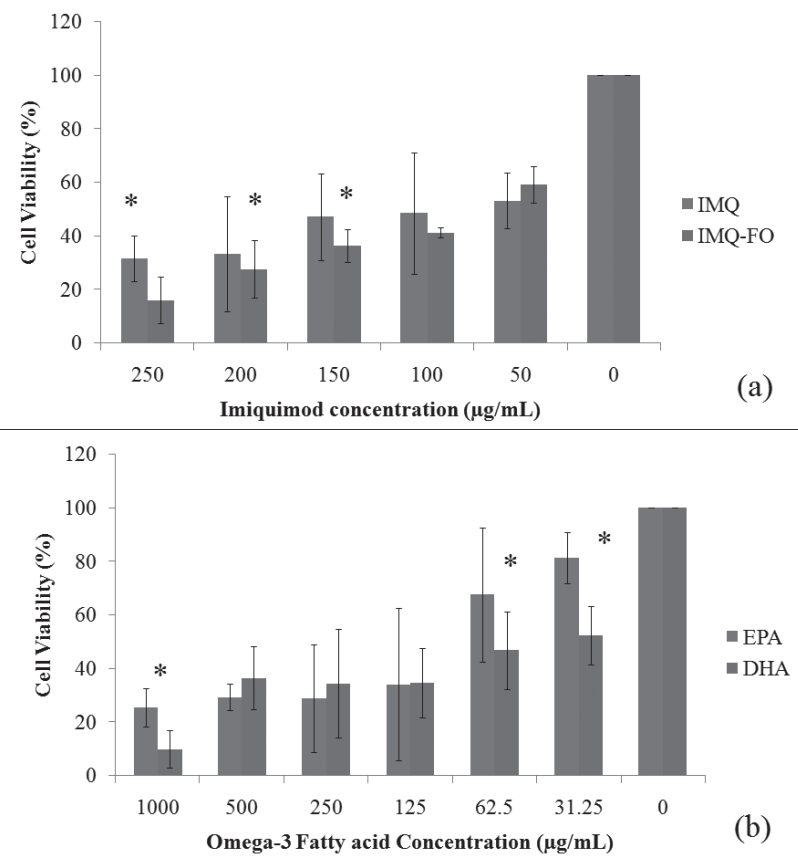

Fig. 1 (a) SCC cell line viability (\%) plot for imiquimod (IMQ) and imiquimod-fish oil (IMQFO) (mean $\pm \mathrm{SD}, \mathrm{n}=3, * p<0.05$, Paired t-test). (b) SCC cell viability (\%) plot for eicosapentaenoic acid (EPA) and docosahexaenoic acid (DHA) (mean $\pm \mathrm{SD}, \mathrm{n}=3$, $* p<0.05$, Paired t-test).

SCC and BCC. The lack of fish oil influence on imiquimod inhibitory activity might be due to the fact that the omega-3 fatty acids in fish oil are present as triglycerides, which might have reduced in their inhibitory activity as observed in pure EPA and DHA alone.

\subsection{Enzyme-linked immunosorbent assay for human skin carcinoma cell lines}

Enzyme-linked immunosorbent assay was conducted to provide an insight into immunomodulatory influence of fish 

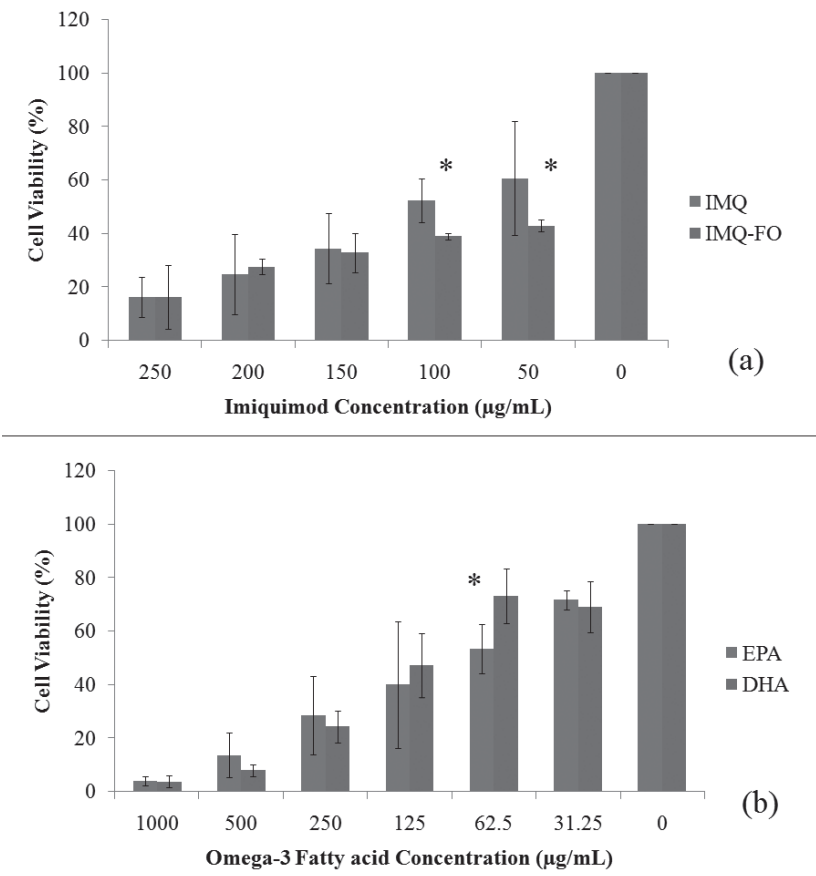

Fig. 2 (a) BCC cell viability (\%) plot for imiquimod (IMQ) and imiquimod-fish oil (IMQ-FO) (mean $\pm \mathrm{SD}, \mathrm{n}=3, * p<0.05$, Paired t-test). (b) BCC cell viability (\%) plot for EPA and DHA (mean $\pm \mathrm{SD}, \mathrm{n}=3,{ }^{*} p<0.05$, Paired t-test).

oil with imiquimod and omega-3 fatty acids on SCC and BCC. It would also help to define the role of fish oil and omega-3 fatty acids in reducing the imiquimod-induced inflammatory side-effects.

\subsubsection{Interleukin-6}

IL6 is known to promote malignant skin growth of squamous cell carcinoma ${ }^{22)}$ and overexpression of IL6 has also been reported in basal cell carcinoma cells ${ }^{23)}$. IL6 expression in SCC and BCC cell lines was determined and concentration-dependent response was plotted for the IMQ and IMQ-FO mixtures. The BCC cells showed higher expression of IL6 than SCC cells. Both IMQ and IMQ-FO mixtures produced concentration-dependent decline in IL6 expression, whereas IMQ-FO treatment resulted in higher inhibition than did treatment with IMQ alone (Fig. 3). To understand the role of omega-3 fatty acids, carcinoma cells treated with EPA and DHA dilutions were also analyzed with immunoassay and it was found that both fatty acids produced a concentration-dependent decline in IL6, whereas DHA exhibited higher inhibition of IL6 than did EPA(Fig. 4). It could be suggested that the omega-3 fatty acids offer a potential for IL6 inhibition in non-melanoma skin carcinoma and greater decline of IL6 production by IMQ-FO mixture compared to that of IMQ alone might again be due to omega-3 fatty acids, especially DHA (Fig. 4).
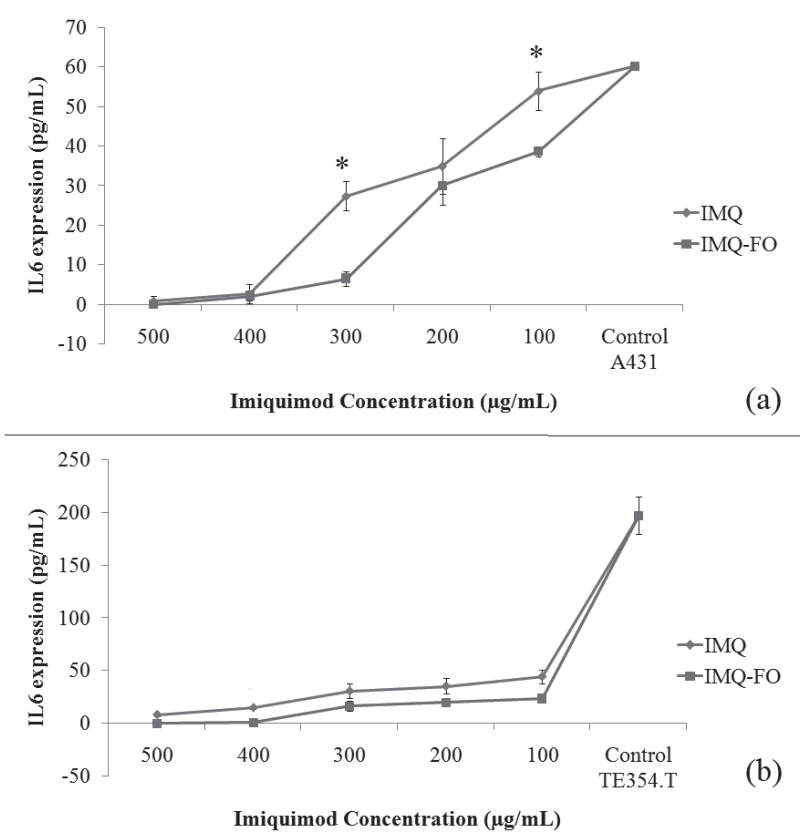

Fig. 3 (a) Concentration-dependent inhibitory effects of imiquimod (IMQ) on interleukin (IL)-6 expressions in SCC cells. Imiquimod-fishoil (IMQ-FO)significantly suppressed IL6 expression compared to IMQ alone or control $(* p<0.05$, Paired t-test). (b) Concentrationdependent inhibitory effect of imiquimod (IMQ) on IL6 expression in BCC cells. Treatment with imiquimod-fish oil (IMQ-FO) markedly suppressed IL6 expression compared to IMQ treatment alone or that observed for the control group $\mathrm{BCC}$, but the difference was not statistically significant.

\subsubsection{Tumor necrosis factor}

TNF- $\alpha$ is an important cytokine known for its pro-inflammatory activity and tumor progression. Therefore, TNF- $\alpha$ expression in SCC and BCC cell lines was determined and concentration-dependent response was plotted for the IMQ, IMQ-FO, and omega-3 fatty acids. Imiquimod is known for its inflammatory side effects ${ }^{3,4}$, and during the study IMQ concentrations appeared to increase the TNF- $\alpha$ expression in both carcinoma cell lines (Fig. 5). Although fish oil is known for its anti-inflammatory activity ${ }^{11,12)}$, but in this case it was unable to significantly reduce the TNF- $\alpha$ expression in cells treated with IMQ-FO mixtures (Fig. 5). The lack of anti-inflammatory activity by fish oil could be due to the fact that the omega-3 fatty acids in fish oil are present in triglyceride form which might have reduced its activity versus pure EPA and DHA. Both omega-3 fatty acids, EPA and DHA, also exhibited concentration-dependent decline of TNF- $\alpha$ expression in SCC and BCC, in which case EPA was appeared to be more effective in 

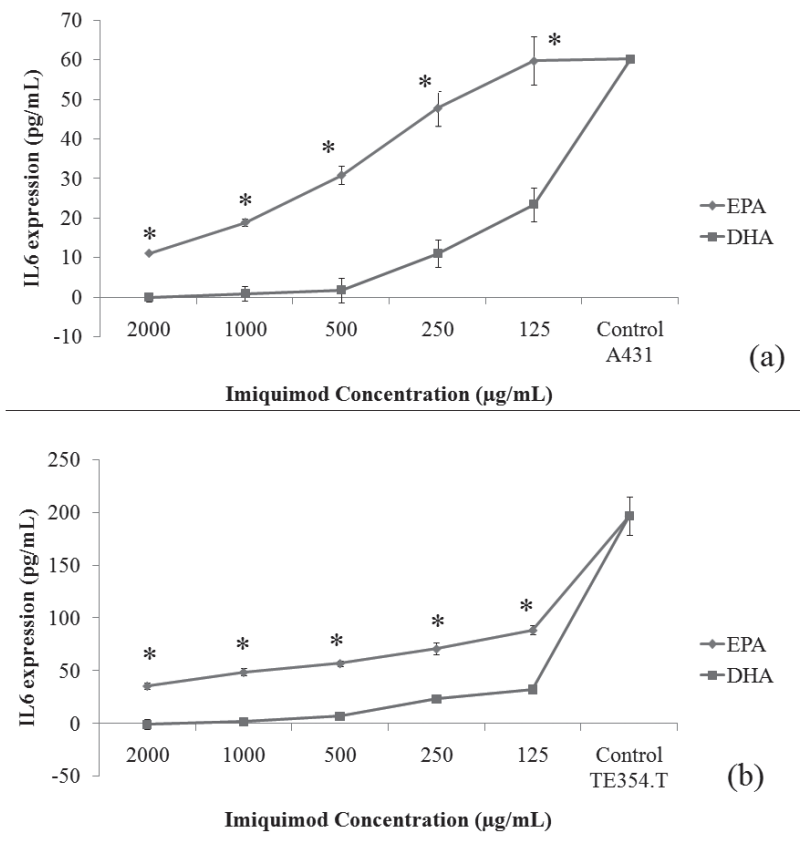

Fig. 4 (a) Omega-3 fatty acids inhibited interleukin (IL)-6 expression in SCC cells in a concentration-dependent manner. Docosahexaenoic acid (DHA) significantly suppressed IL6 expression compared to eicosapentaenoic acid (EPA) treatment alone or control $\left({ }^{*} p<0.05\right.$, Paired t-test). (b) Omega-3 fatty acids inhibited IL6 expression in BCC cells in a concentration-dependent manner. DHA treatment markedly suppressed IL6 expression compared to EPA or to the findings of the control BCC cells $(* p<0.05$, Paired t-test).

TNF- $\alpha$ inhibition compared to DHA (Fig. 6). Localized $\mathrm{TNF}-\alpha$ may cause tumor regression but its excessive systemic presence might increase the risk of cancer development and metastasis ${ }^{24)}$. Therefore, cancer and inflammation treatment has focused primarily on inhibition of TNF- $\alpha^{24,25)}$. The omega-3 fatty acids in fish oil are known for their anti-inflammatory activity and also known to inhibit TNF- $\alpha$ production ${ }^{26,27)}$. Therefore they could prove to be beneficial in treatment against skin carcinoma.

3.3.3 Interleukin-10

IL10 is an anti-inflammatory cytokine and offers an immunoregulatory effects known to downregulate pro-inflammatory cytokines such as IL6 and TNF- $\alpha^{19)}$. Therefore, it has been studied for its antitumor role in skin carcinogene$\mathrm{sis}^{28}$. IL10 expression in SCC and BCC cell lines was determined and concentration-dependent response was plotted for the IMQ and IMQ-FO mixtures. Both IMQ and IMQ-FO produced concentration-dependent increases in IL10 expression, whereas IMQ-FO treatment resulted in greater production of IL10 than did treatment with IMQ (Fig. 7).
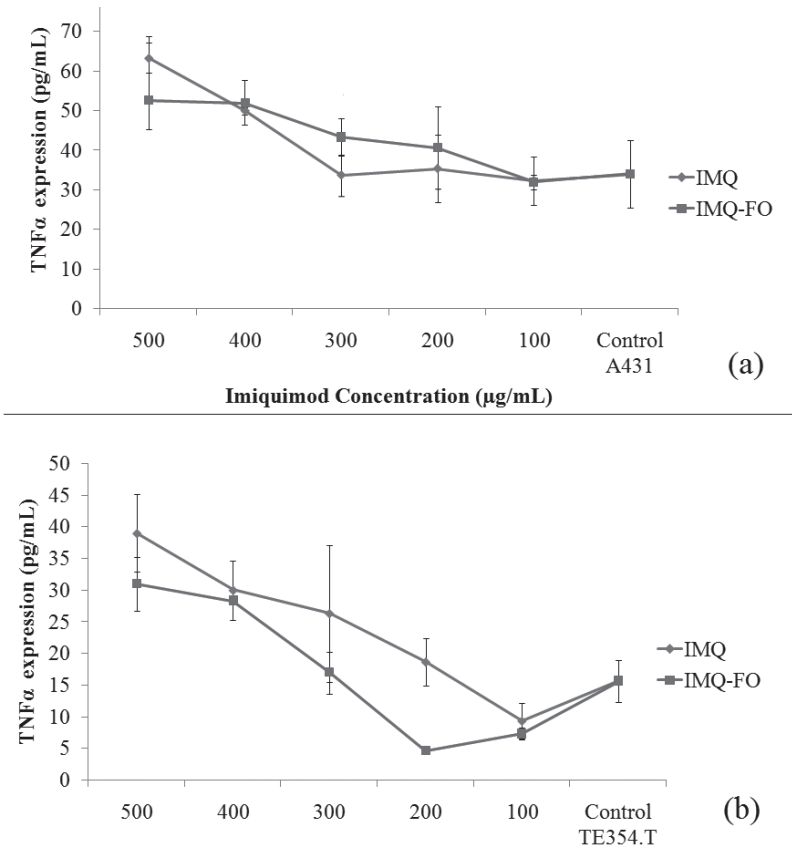

Fig. 5 (a) Concentration-dependent effects of imiquimod (IMQ) on production of TNF- $\alpha$ in SCC cells. The difference in production of tumor necrosis factor (TNF)- $\alpha$ between cells treated with IMQ or imiquimod-fish oil (IMQ-FO) was not significant. (b) IMQ had concentrationdependent effects on the production of TNF- $\alpha$ in BCC cells. The difference in production of TNF- $\alpha$ between cells treated with IMQ or IMQFO was not significant.

To understand the role of omega-3 fatty acids, carcinoma cells treated with EPA and DHA dilutions were also analyzed with immunoassay and it was found that both fatty acids produced a concentration-dependent increase in IL10 production, especially EPA(Fig. 8). In our earlier work, we found that there is an interaction between EPA and imiquimod compound that could help in overcome the imiquimod related inflammatory side-effects ${ }^{5}$. This EPAimiquimod lipophilic-complex forming tendency could also be utilized in treating skin cancer. Imiquimod and omega-3 fatty acids are known to upregulate the production of IL $10^{29,30)}$. The combined influence of IMQ-FO produced a higher expression of IL10 than did IMQ, which could be due to the omega- 3 fatty acids, especially EPA. IL10 is known to increase antitumor immunity and to inhibit tumor-associated inflammation ${ }^{19)}$. Therefore, it can provide a balance between the inflammatory and immunopathologic activities of other Th1 cytokines ${ }^{31}$. EPA has shown more potency in production of IL10 and, with IMQ, it could produce a combined or an additive antitumor effect against non-melanoma skin cancer. 

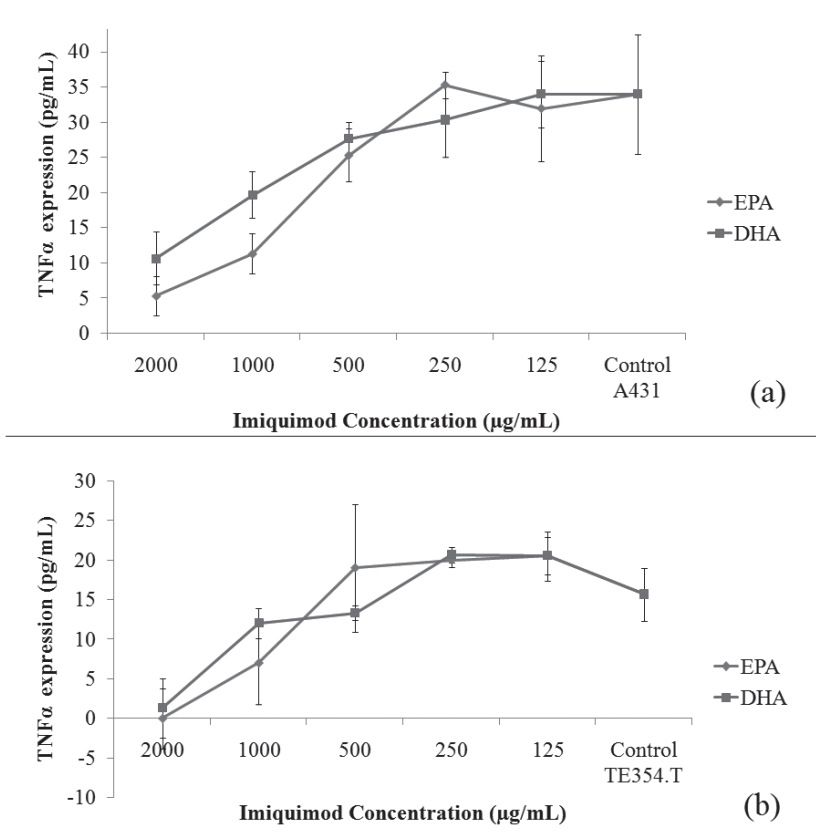

Fig. 6 (a) Omega-3 fatty acids inhibited tumor necrosis factor (TNF)- $\alpha$ production in a concentrationdependent manner in SCC cells. The difference in inhibition of TNF- $\alpha$ between cells treated with eicosapentaenoic acid (EPA) or docosahexaenoic acid (DHA) was not significant. (b) Omega-3 fatty acids had concentration-dependent inhibitory effects on TNF- $\alpha$ in BCC cells. The difference in TNF- $\alpha$ inhibition between cells treated with EPA or DHA was not significant.

\section{Conclusion}

Imiquimod and fish oil are both immune response modifiers and rather than directly killing the carcinoma cells they exert their inhibitory activity by modulating the cell immune response. Imiquimod, imiquimod-fish oil mixture, and omega-3 fatty acids EPA and DHA were monitored for their immunomodulatory role in human non-melanoma carcinoma cell lines. The imiquimod-fish oil and imiquimod alone exhibited similar inhibition of cell growth than did imiquimod alone, and IMQ-FO exhibited significantly higher concentration-dependent immunomodulation both in SCC and BCC cell lines. EPA and DHA also displayed growth inhibition of human skin carcinoma cell lines and immune-modulatory activity proportional to the fatty acids concentration. EPA and imiquimod both stimulate production of IL10, an anti-inflammatory cytokine which increases the potential for an additive or combined action against cancer cells. Omega-3 fatty acids serve as inducers of IL10 and suppressors of IL6, and TNF- $\alpha$, which would not only depress tumor growth but would also serve as an adequate control of the inflammatory response of skin cancer. Thus it can be concluded that an imiquimod-fish oil combination
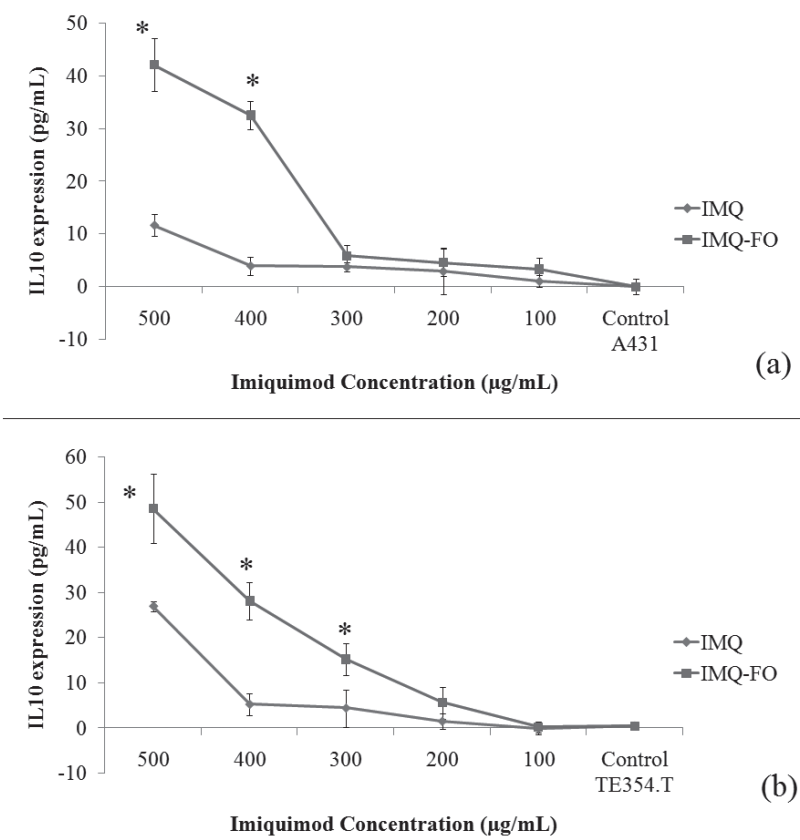

Fig. 7 (a) Concentration-dependent effects of imiquimod (IMQ) on production of interleukin (IL)-10 in SCC cells. Imiquimod-fish oil (IMQFO) treatment upregulated IL10 expression and the difference was significant compared to IMQ treatment outcome and to the findings of the control A431 cells $\left({ }^{*} p<0.05\right.$, Paired t-test). (b) IMQ had concentration-dependent effects on IL 10 production in BCC cells. IMQ-FO treatment increased the expression of IL10 and the difference was significant when compared to IMQ treatment and to the findings of control BCC $\left({ }^{*} p<0.05\right.$, Paired t-test).

can influence inhibition of non-melanoma skin carcinoma cells but further in vivo studies are needed to understand their role in skin cancer. Furthermore, as free EPA and DHA (as opposed to triglyceride forms) were proven to show better immunomodulatory activity against skin carcinoma cell lines as compared to fish oil. Therefore imiquimod should also be studied along with the combination of IMQ-EPA and IMQ-DHA to investigate the omega-3 fatty acid influence on the anti-cancer drug.

\section{Acknowledgments}

The authors would like to thank the Center for Research and Instrumentation, and Faculty of Pharmacy Universiti Kebangsaan Malaysia for providing research grant ERGS/1/2013/SKK02/UKM/02/3 and additional support during this study. 


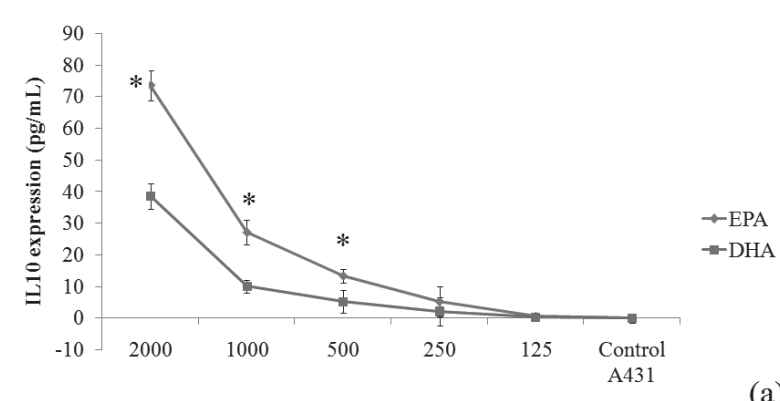

(a)

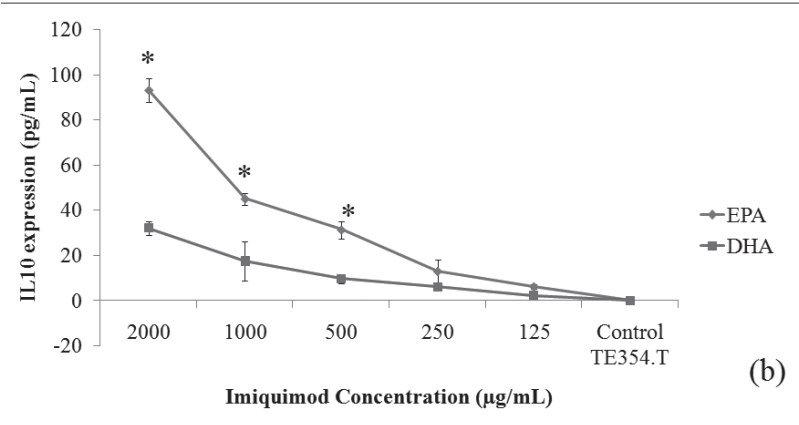

Fig. 8 (a) Concentration-dependent effects of omega-3 fatty acids on interleukin (IL)-10 production in SCC cells. Eicosapentaenoic acid (EPA) upregulated expression of IL10 and the difference was significant compared to docosahexaenoic acid (DHA) treatment outcome and to the findings of control SCC cells $(* p<$ 0.05 , Paired t-test). (b)Omega-3 fatty acids had concentration-dependent effects on production of IL10 in BCC cells. EPA treatment markedly suppressed the expression of IL10 and the difference was significant compared to DHA treatment and to the findings of control $\mathrm{BCC}$ cells $(* p<0.05$, Paired t-test).

\section{Conflicts of interest}

Authors have no conflict of interest to report.

\section{References}

1) Sauder, D. N. Immunomodulatory and pharmacologic properties of imiquimod. J. Am. Acad. Dermatol. 43, 6-11(2004).

2) Teicher, B. A.; Ara, G.; Buxton, D.; Leonard, J.; Schuab, R. G. Optimal scheduling of interleukinc -12 therapy and chemotherapy in the murine MB-49 bladder carcinoma and B-16 melanoma. Clin. Cancer Res. 3, 1661-1667(1997).

3) Cantisani, C.; Lazic, T.; Richetta, A. G.; Clerico, R.; Mattozzi, C.; Calvieri, S. Imiquimod 5\% cream use in dermatology, side effects and recent patents. Recent Pat. Inflamm. Allergy Drug Discov. 6, 65-69 (2012).
4) Rosenblatt, A.; de Campos, G. H. G. Local and systemic adverse effects of imiquimod therapy for external anogenital warts in men: report of three cases. Int. J. STD AIDS 23, 909-910 (2012).

5) Khurram, R.; Aluwi, M. F.; Kamal, R.; Wai, L. K.; Mohd Cairul, I. M. A.; Mohd Hanif, Z. Probing the effects of fish oil on the delivery and inflammation-inducing potential of imiquimod. Int. J. Pharm. 490, 131-141 (2015).

6) Kadir, R.; Stempler, D.; Liron, Z.; Cohen, S. Delivery of theophylline into excised human skin from alkanoic acid solutions: a "push-pull" mechanism. J. Pharm. Sci. 76, 774-779 (1987).

7) Christopher, P. T.; Jamie, P.; Thomas, T.; Charles, M. H. Probing the skin permeation of fish oil/EPA and ketoprofen: 1. NMR spectroscopy and molecular modelling. Int. J. Pharm. 338, 207-212 (2007).

8) Darren, J. H.; Bruce, J. H. Omega-3 fatty acids from fish oils and cardiovascular disease. Mol. Cell. Biochem. 263, 217-225 (2004).

9) Neil, M. I.; Clifford, A. H.; Ayca, G. Omega-3 Fatty Acids for Prevention of Breast Cancer: an Update and the State of Science. Curr. Breast Cancer Rep. 5, 247-254 (2013).

10) Liam, D. C.; Siong-Seng, L.; Ashok, R. V. Chromosome instability and carcinogenesis: Insights from murine models of human pancreatic cancer associated with BRCA2 inactivation. Mol. Oncol. 8, 161-168 (2014).

11) Zulfakar, M. H.; Ong, C. M. Y.; Heard, C. M. The effects of betamethasone dipropionate and fish oil on $\mathrm{HaCaT}$ proliferation and apoptosis. Int. J. Pharm. 434, 399405 (2012)

12) Carmelo, P.; Salvatore, T.; Luisa, R.; Natale, A. S.; Francesco, B. Invitro percutaneous absorptiion studies and invivo evaluation of antiinflammatory activity of essential fatty acids (EFA) from fish oil extracts. Int. $J$. Pharm. 299, 41-48(2005).

13) Naoki, O.; Mariko, F.; Yuriko, N.; Tomoko, F.; Yasuo, T.; Shinasaku, N.; Tadanori, M. A quantitative in vivo method of analyzing human tumor-induced angiogenesis in mice using agrarose microencapsulation and hemoglobin enzyme linked immunosorbent assay. Jpn. J. Cancer Res. 86, 1182-1188(1995).

14) Faust, J. B.; Meeker, T. C. Amplification and expression of bcl-1 gene in human solid tumor cell lines. Cancer Res. 52, 2460-2463 (1992).

15) Janmaat, M. L.; Frank, A. E. K.; Jose, A. R.; Giuseppe, G. Response to epidermal growth factor receptor inhibitors in non-small cell lung cancer cells: Limited antiproliferative effects and absence of apoptosis associated with persistent activity of extracellular signal regulated kinase or akt kinase patheay. Clin. Cancer Res. 9, 2316-2326 (2003).

16) Ruby, J. A.; Manickam, V.; Karunagaran, D. Inhibition 
of $\mathrm{NF}_{-\mathrm{k}} \mathrm{B}$ sensitizes A431 cells to epidermal growth factor induced apoptosis, whereas its activation by ectopic expression of RelA confers resistance. J. Biol. Chem. 278, 25490-25498(2003).

17) Bin, W.; Ji, L.; Damao, H.; Weiwei, W.; Yu, C.; Youxiang, L.; Xiaowei, T.; Hongfu, X.; Faqing, T. Baicalein mediates inhibition of migration and invasiveness of skin carcinoma through ezrin in A431 cells. BMC Cancer. 11, 527-535 (2011).

18) Peh, B. K., RUNX3 acts as an oncogene through a hedgehog-dependent pathway in selected human neoplasms, in Pathology 2011, National University of Singapore: Singapore. p. 134.

19) Michele, W. L. T.; Phillip, K. D.; Mark, J. S. Stable IL10: A new therapeutic that promotes tumor immunity. Cancer Cell 20, 691-693(2011).

20) Susan, J. H.; Dirkjan, H.; George, F. M.; Thomas, S. K.; Adam, W. C.; Ilse, G. M.; Carl, F. S.; Danielle, M. M.; Chrysalyne, S.; Clark, R. A. Imiquimod enhances IFN- $\gamma$ production and effector function of $\mathrm{T}$ cells infiltrating human squamous cell carcinomas of the skin. J. Invest. Dermatol. 129, 2676-2685(2009).

21) Wiltrud, L.; Depner, S.; Sabine, S.; Eva, O.; Nicola, C.; Alexandra, J.; Norbert, E. F.; Margareta, M. M. IL-6 promotes malignant growth of skin SCCs by regulating a network of autocrine and paracrine cytokines. Int. J. Cancer 128, 2803-2814 (2011).

22) Lederle, W.; Depner, S.; Schnur, S.; Obermueller, E.; Catone, N.; Just, A.; Fuseing, N. E.; Mueller, M. M. IL-6 promotes malignant growth of skin SCCs by regulating a network of autocrine and paracrine cytokines. Int. J. Cancer 128, 2803-2814 (2011).

23) Shiou-Hwa, J.; Shing-Chuan, S.; Hsien-Ching, C.; WeiLing, T.; Kuo, M.-L. Overexpression of interleukin-6 in human basal cell carcinoma cell lines increases antiapoptotic activity and tumorigenic potency. Oncogene
20, 198-202 (2001).

24) Fiorella, G.; Patrizia, F.; Raffaele, P.; Ilaria, P.; Vincenzo, F.; Mario, R. TNF/VEGF cross-talk in chronic inflammation-related cancer initiation and progression: An early target in anticancer therapeutic strategy. In Vivo 21, 147-162 (2007).

25) $\mathrm{Wu}, \mathrm{Y}$.; Zhou, B. P. TNF- $\alpha / \mathrm{NF}_{-\mathrm{k}} \mathrm{B} /$ Snail pathway in cancer migration and invasion. Br. J. Cancer 102, 639$644(2010)$.

26) Caterina, D. R.; Cybulsky, M. I.; Clinton, S. K.; Gimbrone, M. A. J.; Libby, P. The omega-3 fatty acid docosahexaenoate reduces cytokine-induced expression of proatherogenic and proinflammatory proteins in human endothelial cells. Arterioscler. Thromb. Vasc. Biol. 14, 1829-1836 (1994).

27) Philip, C. C. Omega-3 fatty acids and inflammatory process. Nutrients 2, 355-374(2010).

28) Karin, L.; Jenny, A.; Maik, V.; Mariette, M.; Sandra, B.; Thomas, S.; Stephan, G.; Stefan, B. IL10 controls ultraviolet-induced carcinogenesis in mice. J. Immunol. 179, 365-371 (2007).

29) Kemeny, L., Non-surgical treatment of keratinocyte skin cancer. Topical Imiquimod, ed. Gregor, B. E. J., Donald, M., and Lajos, K. Berlin-Heidelberg, Springer (2010).

30) Anke, J.; Michael, G.; Bernhard, W.; Christina, N.; Alexander, R.; Alfred, L.; Dagmar, B.; Holger, G.; Michael, R.; Gerhard, J. Evaluation of suppressive and pro-resolving effects of EPA and DHA in human primary monocytes and T-helper cells. J. Lipid Res. 54, 923935 (2013).

31) Yone, V. N. C.; Maria, C. A. B.; Juliana, K. d. A. L. N.; Jose, C. F.; Valeria, R. A. P. Role of TNF-alpha, IFNgamma, and IL-10 in development of pulmonary tuberculosis. Pulm. Med. 2012, 1-10 (2012). 